\title{
165 MUSIC AND VIDEO: MEANS TO SUCCESS
}

N Vijay Mohan, A Pallavi Department of Physical Education, Andhra University College of Engineering, Visakhapatnam, Andhra Pradesh, India

\subsection{6/bjsm.2010.078725.165}

Young people are typically confident in the use of the latest technologies and because many aspiring athletes are from this age range, sport psychologists and coaches should consider using this technology in their work. A key question for applied sport psychology practitioners and coaches, therefore, is what technologies will be used by and beneficial for athletes? Here, we deal with MP3 technology to enhance performance. The use of MP3 technology by athletes is often seen as an integral part of mental preparation. Typically, music folders are arranged on an MP3 player to contain music of different genres. However, we assist athletes in developing music folders with specific purposes. For example, folders may be established containing music that is intended to relax, motivate energies, develop confidence or a combination of these. The advent of video MP3 players has introduced further possibilities for the use of technology among sport and exercise psychologists, coaches and athletes. Within our practice, we use video iPod players to reinforce correct technique, direct attention to key goals/objectives and motivate athletes. For example, images of the athlete performing can be merged with images of the role models they aspire to beat. Nowadays as computer technology becomes more sophisticated the ability to produce videos with associated music becomes easier. Athletes value technology and coaches can build upon this by demonstrating how it can be used effectively to enhance performance. 